\title{
Auf dem Weg zum digitalen homo vitruvianus? Medizinisches Selftracking und digitale Gesundheitsanwendungen (DiGA) zwischen Empowerment und Kontrollverlust
}

\author{
Florian Funer
}

Eingegangen: 21. September 2020 / Angenommen: 30. Oktober 2020 / Online publiziert: 8. Dezember 2020

(C) Der/die Autor(en) 2020

Zusammenfassung Zunehmend gewinnen Health Apps an Bedeutung für eine präventive und eigenverantwortliche Ausrichtung des Gesundheitssystems. Die meisten dieser digitalen Gesundheitsanwendungen (DiGA) basieren derzeit auf sog. Selftracking-Technologien, mit deren Hilfe physiologische und psychische Daten sensorgestützt aufgezeichnet und diese zumeist um personalisierte Alltagsinformationen ergänzt werden. Die digitalen Entwicklungen dieser Art lösten in den letzten Jahren eine intensive und deutlich polarisierte Debatte über die Chancen und Gefahren von gesundheitlichem Selftracking aus. Ziel dieser Arbeit ist es, nach einem kurzen Überblick über das Feld des medizinischen Selftracking den polarisierten Diskurs zunächst anhand seiner entscheidenden individuellen und gesellschaftlichen Potenziale zu systematisieren und zu prüfen. Es stellt sich heraus, dass Selbstvermessungstechniken keineswegs ein neues Phänomen, sondern vielmehr seit langem fester Bestandteil medizinischen Wirkens in Diagnostik und Therapie sind. Als für die Implementierung von Health Apps in die medizinische Regelversorgung besonders bedeutend wird das Spannungsfeld zwischen individuellem Empowerment und potentiellem Kontrollverlust der Nutzenden herausgestellt. Abschließend werden mögliche Implikationen für die aktuelle Ausgestaltung des neuen Digitale-Versorgung-Gesetzes (DVG) aufgezeigt und auf einige bestehende ,blinde Flecken“ hingewiesen.

Schlüsselwörter Medizinisches Selftracking · Digitale Gesundheitsanwendungen (DiGA) · Empowerment · Selbst- und Fremdbestimmung · Digitale-VersorgungGesetz (DVG)

Die vorliegende Arbeit wurde mit dem Nachwuchspreis 2020 der Akademie für Ethik in der Medizin e. V. ausgezeichnet.

Dr. med. F. Funer, Mag. theol., M.A. ( $₫)$

Lehrstuhl für Moraltheologie/Theologische Ethik, Katholisch-Theologische Fakultät,

Eberhard-Karls-Universität Tübingen, Tübingen, Deutschland

E-Mail: florian.funer@uni-tuebingen.de 


\title{
On the way to the digital homo vitruvianus? Medical self-tracking and digital health applications (DiGA) between empowerment and loss of control
}

\begin{abstract}
Definition of the problem Health Apps are becoming increasingly important for a preventive and responsible orientation of the health system. Currently, most of these digital health applications (DiGA) are based on so-called self-tracking technologies which record physiological and psychological data via sensors, usually combined with personalized everyday information. In the last few years, these digital developments have launched an intense and clearly polarized debate about the opportunities and dangers of self-tracking in healthcare.

Arguments After a brief overview of medical self-tracking, this essay wants to systematize and check the polarized discourse on the basis of its decisive individual and social potentials. It turns out that self-measurement techniques have long been an integral part of medical practice in diagnostics and therapy.

Conclusion The area of tension between individual empowerment and potential loss of control by the users will be emphasized as being especially important for the implementation of health Apps in primary health care. Finally, possible implications for the current design of the new German Digital Care Law (Digitale-VersorgungGesetz) are shown and some existing "blind spots" are highlighted.
\end{abstract}

Keywords Medical self-tracking · Digital health applications · Empowerment · Autonomy and heteronomy · German Digital Care Law

\section{Einführung - Von der Vermessung des Menschen ...}

Auf der Suche nach der natürlichen und gottgegebenen Gesetzmäßigkeit in den anatomischen Proportionsverhältnissen wetteiferten die berühmtesten Künstler der Renaissance um eine möglichst formvollendete Darstellung des Menschen. Unter den diversen Umsetzungen tat sich Leonardo da Vincis homo vitruvianus, sein vitruvianischer Mensch, in besonderer Weise hervor und entwickelte sich als eine der bekanntesten menschlichen Proportionsstudien weltweit bis heute zu einem Symbol für Symmetrie, Ästhetik und Körperbewusstsein. ${ }^{1}$ Doch nicht erst das wachsende Interesse der Renaissance-Künstler im 15. und 16. Jahrhundert bot jenes Substrat für die Suche nach und die Faszination von einer Perfektion des menschlichen Körpers und seiner Abläufe, griffen auch sie für ihre Werke auf Vorstellungen der antiken Hochkultur eines gymnastisch-athletisch definierten Körpers als Ausdruck der Schönheit der Schöpfung zurück. ${ }^{2}$ Die anatomischen Harmonieprinzipien, die

\footnotetext{
1 Leonardo da Vinci, Der vitruvianische Mensch, ca. 1490, Tinte auf Papier, 34,4×24,5 cm, Galleria dell' Accademia Venedig.

2 Vgl. die namensgebende Quelle für Leonardos Umsetzung Vitruv (1964, 3, 1, 1, S. 138), in welcher eine Lehre des wohlgeformten Körpers (homo bene figuratus) und der zugrundeliegenden Prinzipien der Ästhetik entfaltet wird.
} 
etwa der antike Autor Vitruv anfanghaft theoretisch reflektierte, kulminierten in der mittelalterlichen Rezeption in zunehmend detaillierteren Vermessungen und Proportionsbeobachtungen des Menschen mit dem Ziel der Gotteserkenntnis (Zimmermann 1984). Vom homo bene figuratus Vitruvs seien, ihrer Auffassung nach, alle Maße abzuleiten und in ihm alle Zahlenverhältnisse zu finden - das Projekt der Vermessung des Menschen, der Anthropometrie, fand ihren entscheidenden Impuls und sollte bis in die Gegenwart zahlreiche Transformationen erfahren.

Die Erkenntnis, dass angesichts des breiten Variationsspektrums des menschlichen Körperbaus keine festen anatomischen Verhältnisgrößen existieren, erbrachten anthropologische Studien bereits Ende des 20. Jahrhunderts (Flügel et al. 1986). Ungeachtet dieses Umstandes verebbten die Faszination der Selbstvermessung und die Vorstellung einer menschlichen „Norm“ keineswegs, sondern gewannen kraft der zunehmenden technischen Entwicklungen und damit einhergehenden Möglichkeiten einer Vermessung des Menschen vielmehr an Fahrt. Eine zunehmende Konvergenz verschieden gearteter, aktiv auf Optimierung ausgerichteter Maßnahmen, wie die Diätetik und das Body-Building, einerseits und der zunehmenden Suche, mittels immer tiefgreifenderer Analysen der Anatomie und Physiologie, nach einer in anthropologischen „Normen“ bereits angelegten Perfektion andererseits strebt mit zunehmender Geschwindigkeit einem körperkultischen Idealtypus entgegen. Das Streben nach einer potenziell irgendwann oder aktuell schon erreichbaren Perfektion begleitet die Geschichte der Menschheit, wie geschildert, seit jeher und wird dies voraussichtlich auch in Zukunft weiterhin tun. Die konkrete Ausgestaltung unterliegt aber aufgrund der steigenden technischen und medizinischen Möglichkeiten sowie der damit schrittweise einhergehenden Umsetzbarkeit der vermeintlichen Perfektion einer besonderen gesellschaftlichen Verantwortung.

Der in den letzten Jahren anschwellende Markt sogenannter Health Apps bzw. digitaler Gesundheitsanwendungen (DiGA) für das Smartphone, deren Kosten nach Aufnahme in das Verzeichnis des Bundesinstituts für Arzneimittel und Medizinprodukte (BfArM) seit Dezember 2019 auch von den gesetzlichen Krankenkassen übernommen werden ${ }^{3}$, etabliert sich zunehmend als lukratives Geschäft Softwareentwickelnder Unternehmen. Health Apps haben insofern die medizinische Regelversorgung erreicht, noch ehe ihre nähere rechtliche Regulierung einen Abschluss gefunden hat. Diese Entwicklung unterstreicht das in ihnen vermutete Potenzial einer Verbesserung des gesellschaftlichen Gesundheitsniveaus und eines Abbaus strukturell bestehender Defizite durch einen gesteigerten Daten- und Informationsfluss. Hoffnungen auf eine durch Selbsterkenntnis gesteigerte Eigenverantwortung und Selbstbestimmung in der Gestaltung der eigenen Gesundheit - oftmals verkürzt unter dem Begriff des Empowerment angeführt - treiben gerade angesichts der gesundheitsdemographischen Zukunftsaussichten eine Expansion des Markts von DiGA an. Neben diesen Potenzialen auf positiver Seite illustrieren einige, insbesondere soziologische Arbeiten der letzten Jahre, die sich mit dem Phänomen des sog. Selftracking, hier noch zumeist mithilfe von Fitnessarmbändern u.ä., auseinan-

\footnotetext{
3 Das Gesetz für eine bessere Versorgung durch Digitalisierung und Innovation (,Digitale-VersorgungGesetz“, DVG) ist seit dem 9. Dezember 2019 neuer Bestandteil des Sozialgesetzbuchs (SGB) V; vgl. Bundesgesetzblatt (2019), I, Nr. 49, S. 2562-2584.
} 
dersetzten (Mämecke et al. 2018; Deutscher Ethikrat 2017; Duttweiler et al. 2016; Abend et al. 2016; Selke 2016b), mögliche Gefahren einer ,Verobjektivierung“ des Individuums (Villa 2012; Zillien et al. 2015) und einer zunehmenden gesellschaftlichen „Medikalisierung“ (Sharon 2017; Maturo und Moretti 2018). Entgegen der Annahme einer Stabilisierung und Förderung der Selbstbestimmung werden vor allem im möglichen Verlust persönlicher Daten an Dritte (Big Data) (Gethmann 2018; Amunts et al. 2018; UNESCO-ICB 2017; Deutscher Ethikrat 2015, 2017; Mainzer 2014; Stampfl 2013) sowie dem fortwährenden Optimierungsimpetus der Nutzenden Momente der Fremdbestimmung erkannt.

Wenngleich die Debatte angesichts der Zuschreibungen an DiGA kaum deutlicher durch Polarisierungen charakterisiert werden kann, müssen dennoch gangbare Konsequenzen für die derzeitige Ausgestaltung einer Praxis folgen. So wird in dieser Arbeit ein zweifaches Ziel verfolgt: Auf der einen Seite bedürfen Entscheidungen hinsichtlich einer Integration anthropometrischer Anwendungen in die Gesundheitsversorgung einer ausgewogenen Darstellung ihres Für und Widers. Nach einer Skizzierung der aktuellen Möglichkeiten von DiGA sollen daher die bereits geführten Diskurse verschiedener Disziplinen systematisch zusammengeführt werden. Ein besonderer Fokus wird hierbei auf die Potenziale im Sinne eines Empowerment sowie dessen Grenzen hin zum Kontrollverlust gelegt. Auf der anderen Seite sollen die hier dargestellten und gebündelten Erkenntnisse einer Überprüfung des aktuellen Digitale-Versorgung-Gesetzes (DVG) sowie dessen noch ausstehender Modifikationen dienen. Dabei sollen sowohl die bereits berücksichtigten Aspekte des Diskurses gewürdigt als auch auf noch bestehende ,blinde Flecken“ hingewiesen werden, die sich auf die konkrete Implementierung des medizinischen Selftracking bzw. von DiGA in die therapeutische Patientenbegleitung beziehen.

\section{Medizinisches Selftracking und DiGA}

Das digitale Tracking medizinischer Informationen erwuchs im Wesentlichen aus den Entwicklungen der letzten beiden Jahrzehnte: auf der einen Seite als Instrumente der (prä-)klinischen Forschung und Diagnostik, bspw. klassisch als Langzeitaufzeichnungen elektrischer Herzaktivität und des Blutdrucks oder etwa zur Früherkennung von Gangparameterveränderungen bei neurodegenerativen Erkrankungen, sowie auf der anderen Seite in avantgardistischen Kreisen des Sport- und Fitnessbereichs zur Identifikation von Potenzialen einer Leistungssteigerung (Deutscher Ethikrat 2017; Vormbusch 2016; Duttweiler und Passoth 2016). Während im ersten Anwendungsgebiet der Erkenntnisgewinn aus (patho-)physiologischen Daten vorrangig einer möglichst frühzeitigen Diagnosestellung und individuellen Therapiebegleitung bei Vorliegen von Erkrankungen dienen soll (sog. „Medizin-Apps“), werden in Letzterem der intra- und intersubjektive Vergleich sowie eine Optimierung der physiologischen Abläufe angezielt (sog. „Health Apps“). Beide Formen der Vermessung und Verdatung körperbezogener Informationen werden durch die zunehmende „Smartness“ technischer Innovationen - d.h. klein, mobil, unauffällig, intuitiv, vernetzt zu sein - immer alltagstauglicher, erlauben etwa die Kameraund Tonausstattung, die Mikrosensorik und Internetfähigkeit von Smartphones und 
immer erschwinglichere Messgeräte eine automatische, kontinuierliche und ,immer differenziertere Messungen am eigenen Leib“ (Duttweiler und Passoth 2016, S. 18). Auch ist der:die Tracker:in nicht mehr angewiesen auf die Evaluation durch einen Arzt oder Fitnesstrainer, vielmehr stehen ihm dank exorbitanter Datenmengen im Netz zahlreiche Evaluationskriterien zur eigenhändigen Verfügung. Die ehemals trotz ihres gemeinsamen Messobjektes weitestgehend durch ihre Intention getrennten Anwendungsbereiche diagnostisch-klinischer Unterstützung und sportlich-wettbewerblicher Verbesserung verschwimmen aufgrund der einfacheren Zugänglichkeit und Alltäglichkeit der selbstvermessenden Technologien zusehends. Beide Anwendungsbereiche nähern sich aufgrund der Multifunktionalität der Anwendungen einander an und erschweren daher eine Differenzierung von Medizin- und GesundheitsApps.

Heutiges Selftracking 4 umfasst vielfältige Formen der digitalen Erhebung, Sammlung, Zusammenführung, Visualisierung, Evaluation und Vernetzung gesundheitsbezogener Daten, von Verhaltensweisen (Nahrungsaufnahme, Schlafrhythmus, Arbeitsproduktivität, Internetnutzung oder Kaffeekonsum) über physiologische Zustände (z. B. Herzfrequenz, Blutdruck, Blutzucker, Menstruationszyklus) bis hin zu körperlichen Leistungen (tägliche Schritte, Dauer und Strecke von Lauf- und Fahrstrecken, Anzahl von Fitnessübungen); teilweise werden diese ergänzend verknüpft mit Informationen über psychisch-emotionale Zustände (subjektive Stimmungen, Glückserfahrungen, Ausdrucksweisen) (Rode 2019; Selke 2016b; Duttweiler und Passoth 2016; Maturo und Moretti 2018; Danesi et al. 2018; UNESCO-IBC 2017). Es ist daher als eine „,komplexe empirische Erscheinung“ zu verstehen, ,unter deren Namen sich verschiedene Ziele, verschiedene Praktiken und verschiedene Gegenstände der Vermessung vereinen“ (Duttweiler und Passoth 2016, S. 10). TrackingAnwendungen stellen die weite Mehrheit der zurzeit erhältlichen DiGA dar, deren Ziele in der Förderung von Gesundheit sowie in der Unterstützung bei der Erkennung, Überwachung und Behandlung von Krankheiten liegen. Jeder willigen Person bietet sich somit die Möglichkeit, eine Anwendung zu erhalten, „die verspricht, besser zu schlafen, besser zu essen, besser zu atmen, sich besser zu fühlen oder besser zu arbeiten“ (Wiedemann 2016, S. 293). ${ }^{5}$ Zahlreiche Weiterentwicklungen, die diese Daten mit interaktiven Patient:innenplattformen (z.B. PatientsLikeMe, CureTogether, Tu Diabetes), elektronischen Tagebüchern (vgl. DiabetesTagebücher wie SiDiary oder Medikationspläne wie MyTherapy) oder Diagnostikund Therapiemöglichkeiten (vgl. Muttermal-Apps wie SkinVision oder ärztliche Online-Beratungen wie HealthTap, MedHelp oder MDJunction) verknüpfen, lassen

\footnotetext{
4 Auch die Vielzahl an genutzten Begriffen verdeutlicht die Heterogenität des Feldes, so werden etwa im englischsprachigen Bereich mobile health, mHealth, digital health, quantified self, personal informatics nahezu synonym verwandt (Wiedemann 2016; Elsden und Kirk 2014).

5 Ein Einblick in die Bandbreite und Vielzahl dieser Versprechen bietet bereits ein kurzer Blick in die Beschreibungen der bestbewerteten Gesundheits-Apps des Apple-Stores. Oftmals wird die Funktion der Apps bereits anhand ihres Namens deutlich, z. B. Sleep Cycle, Fooducate, My Diet Coach, Waterlogged, Moodscope, Happify, Headspace, Fitplan, MyFitnessPal oder - wohl am deutlichsten - Fitocracy. Vermeintliche Rundum-Sorglos-Apps, wie Simple Symptom Tracker, Nudge und Lark, umfassen diverse Trackingfunktionen über Wohlbefinden, Schlaf, Ernährung und Aktivität und werden daher als ,24/7 Health Coach“ beworben.
} 
den Horizont des ursprünglichen Selftracking überschreiten. ${ }^{6}$ Die Vielfalt von Selftracking-Technologien sowie deren Verknüpfungen mit weiteren Funktionen führt zur Überlagerung präventiver und kurativer Motive der Verbraucher:innen, die eine Differenzierung bei der Bewertung derartiger Anwendungen erfordern.

Heutigen Schätzungen des Bundesverbands für Medizintechnologie (BVMed) zufolge stehen den Nutzenden weltweit rund 150.000 DiGA dieses heterogenen und nahezu unüberschaubaren Feldes zur Verfügung. Umfragen zur Nutzung von Health Apps verdeutlichen, dass das expansive Angebot deutlich von einer steigenden Nachfrage angekurbelt wird. So zeigen jüngste repräsentative Verbraucherbefragungen des IT-Branchenverbands Bitkom bei über 16-Jährigen im Jahr 2019, dass bereits zwei Drittel aller Smartphone-Besitzer:innen Health Apps nutzten. Hierbei würden rund $52 \%$ der Befragten Apps zum Selftracking verwenden - der damit meistgenutzten Funktion unter den DiGA. Darüber hinaus stünden Apps mit Informationen (51\%) und zum Workout (43\%) im Vordergrund (Bitkom Research 2019). Zahlreiche der Nutzenden würden vom Gebrauch profitieren, gaben etwa $53 \%$ an, sie hätten so ihr Training optimieren können, 46\% würden sich mehr bewegen, $44 \%$ wüssten besser über ihren Gesundheitszustand Bescheid, $34 \%$ ernährten sich gesünder und 29\% hätten ihr Gewicht reduzieren können (Bitkom Research 2019; vergleichbare Studien: Depper und Howe 2017; Didžiokaite et al. 2018; Fotopoulou und O'Riordan 2017). Darstellungen negativer Effekte blieben hierbei aus; inwiefern diese überhaupt erhoben wurden, bleibt fraglich.

\section{Selbstquantifizierung in der Medizin - Systematisierung einer polarisierten Debatte}

Angesichts der Heterogenität von DiGA hinsichtlich ihrer Zweckbestimmung, ihrer jeweiligen Umsetzung sowie des tatsächlichen Outcomes bedarf die folgende Analyse positiver und negativer Potenziale von Health Apps einer inhaltlichen Eingrenzung. Im Fokus der Auseinandersetzung sollen daher Anwendungen stehen, die zumindest als wesentlichen Bestandteil Selftracking-Funktionen beinhalten - die derzeitig größte Sparte von DiGA hinsichtlich ihrer Nutzung. Solche, die der reinen Information oder der Kommunikation mit medizinischen Expert:innen dienen, sollen folgend außer Acht gelassen werden.

Die polarisierte Debatte infolge eines Appells Wolfs, dem Begründer der Quantified-Self-Bewegung, im Jahr 2010 zur ,Selbstkontrolle durch Selbsterkenntnis mittels Zahlen“ (Wolf 2010, zitiert in Rode 2019) brachte einerseits positive Reaktionen für die zukünftige Gestaltung gesundheitlicher Versorgung in der medizinischen und Public Health-Literatur (Harrison et al. 2011; Steinhubl et al. 2013; Rabin und Bock 2011; Laakso et al. 2011), andererseits kritische Deutungen hinsichtlich der sozialen, subjektbezogenen und kulturellen Konsequenzen innerhalb der sozialwissenschaftlichen Literatur hervor (Maturo und Moretti 2018; Zillien et al. 2015; Jethani 2015; Lupton 2016, 2014, 2013; Nafus und Sherman 2014; Ruckenstein

\footnotetext{
${ }^{6}$ Selke nutzt hierfür den Begriff Lifelogging, der Selbstvermessung und Lebensprotokollierung verknüpft (Selke 2016b).
} 
2014; Till 2014; Whitson 2013). Folgend sollen beide Seiten des Diskurses anhand dreier miteinander verknüpfter Spannungsfelder zusammengefasst und systematisiert werden: 1.) anhand des Spannungsfelds zwischen empirischem Erkenntnisgewinn durch medizinisches Selftracking und einer befürchteten „Verobjektivierung“ des Menschen, 2.) des Spannungsfelds einer gesamtgesellschaftlich angezielten präventiven Grundhaltung und der Gefahr einer zunehmenden „Medikalisierung“ des Alltags und 3.) des - für die derzeitigen Gestaltungen für am fruchtbarsten gehaltenen - Spannungsfelds zwischen einem potenziellen individuellen Empowerment und einem möglichen Kontrollverlust des Patienten.

\section{Empirischer Erkenntnisgewinn oder Verobjektivierung des Menschen?}

Gegenüber den bisherigen (Selbst-)Vermessungstechniken zeichnen sich DiGA mit „smarter“ Selftracking-Technologie durch eine weitaus kontinuierlichere und umfangreichere Datengenerierung aus. Durch verschiedenartige Sensoren und die weitgehend unsichtbare Implementierung in den Alltag können Informationen über die Anwender:innen gesammelt werden, die deren Bewusstsein bisher nicht vollständig oder aber nur ,subjektiv verzerrt“ zugänglich waren (Schaupp 2016, S. 73). Eine derartige - natürliche - Selektivität der Wahrnehmung und des Bewusstseins wird durch trackende Technologien ausgeglichen und ermöglicht durch die stetige Verkleinerung von Untersuchungsintervallen bis hin zu einer zur Realität nahezu simultanen Aufzeichnung eine bis vor kurzem unmögliche Ausführlichkeit der Dokumentation, Analyse und Evaluation. Nahezu alle Selftracking-Praktiken bedienen sich hierfür einer Visualisierung bisher unsichtbarer Informationen (Reichert 2016). Kritiker:innen einer solchen Praxis betonen, eine auf diese Weise durch Zahlen suggerierte Objektivität und Exaktheit werde allzu schnell als die ,,authentischere“ Wirklichkeit (Pantzar und Ruckenstein 2015, S. 103; Duttweiler und Passoth 2016) interpretiert und Körper- und Selbstwahrnehmung daher ,,zusehends mit Objektivitätsansprüchen überzogen“ (Wiedemann 2016, S. 294; vgl. auch Unternährer 2016). Um dies zu erreichen, würden durch die Quantifizierung des Menschen Kategorien und Verfahren erzeugt, die die Pluralität seines Alltags und seiner Lebensführung vergleichbar zu machen versuchen (Vormbusch 2016); mit sich selbst wie auch mit anderen. Durch die Beobachtung und Orientierung an vermeintlich deskriptiven Grenz- und Mittelwerten kann diesen Kategorien und Verfahren jedoch eine erstaunlich normative Wirkung zuteilwerden. Je höher die Objektivität des Lebens zu sein scheint und je größer das den Daten zugrundeliegende Kollektiv an Individuen wird, desto deutlicher kann sich ihre normierende Wirkung entfalten (Link 2006), sofern die Nutzenden ihre Individualität an den - bereits so benannten - „Normwerten“ auszurichten versuchen. Sich psychologisch einer solchen normativen Kraft der Masse zu entziehen, kann insbesondere angesichts zunehmender technischer und therapeutischer Optionen der Veränderbarkeit alsbald zur Herausforderung werden, die die Kompetenzen und die Persönlichkeiten der Einzelnen übersteigt.

Wie bereits eingangs am Beispiel des homo vitruvianus und seiner antiken Vorbilder deutlich wurde, begleitet die Faszination an der Analyse und einer vermeintlichen „Norm“ des Menschen die Menschheitsgeschichte bereits seit vielen Jahrhunderten. Selbstverständlich verändern die soziokulturellen und soziotechnischen Innovatio- 
nen auch Körper- und Subjektverhältnisse des Menschen fortwährend; hierin einen Anlass zu allzu entwicklungskritischen Dystopien einer „Entleiblichung“ oder gar einer „Auflösung des Selbst in Körperdaten“ (etwa in Duttweiler und Passoth 2016, S. 24) zu erkennen, scheint angesichts der schrittweisen historischen Entwicklungen von der metrischen Vermessung in der frühen Neuzeit über akribische Gewichtsaufzeichnungen seit dem 16. Jahrhundert bis hin zu detaillierten Kalorientabellen im den letzten Jahrzehnten als wenig hilfreich und äußerst fraglich. SelftrackingTechnologien scheinen vielmehr als Form der Stunde eine Möglichkeit zu bieten, auf den Körper und dessen Zustand zu reflektieren, hieraus Wissen zu generieren und so für die meisten Nutzenden eine intensivere Auseinandersetzung mit ihrem Verständnis von „Selbst“ zu eröffnen.

Unmittelbar mit der Datengenerierung und dem dadurch ermöglichten Vergleich der Daten untereinander ist auch die Frage nach dem Datenaustausch und den jeweiligen Adressat:innen verknüpft. So ist der durch die technischen Entwicklungen verursachte potenzielle Nutzen für die Forschung immens. Detaillierte Datenaufzeichnungen, die auch außerhalb klinischer Versuchsszenarien und daher mit reduzierten unerwünschten Testeffekten durchgeführt werden, sind nicht erst durch Selftracking ermöglicht worden. Die Vielzahl weltweit mittels Health Apps generierter Daten lässt jedoch durch gigantische Studienpopulationen und die Breite der gleichzeitig erfassten Parameter auf Beobachtungs- und Interventionserkenntnisse mit höchster statistischer Signifikanz hoffen (Paton et al. 2012; Sharon 2017; Vormbusch 2016; Deutscher Ethikrat 2017). Vernetzungen mit personalisierten Informationen, wie soziokultureller Herkunft, genetischer Prädisposition, persönlicher Lebensführung und privatem Konsum, steigern das Potenzial selbstvermessener Daten zusätzlich. Medizinisches Selftracking könnte insofern einen enormen, bisher innerhalb der klinischen Forschung unmöglichen Beitrag zum wissenschaftlichen Erkenntnisgewinn leisten. ${ }^{7}$

So groß die Potenziale wissenschaftlicher Erkenntnis sein mögen, wird mit der Zunahme von Informationen jedoch auch das Interesse ökonomischer Akteure gesteigert. Beispielhaft sind hierbei US-amerikanische Krankenversicherungen zu nennen, die ihren Versicherten einen rabattierten Beitragssatz anboten, sofern diese freiwillig Selftracking-Informationen mit dem Unternehmen teilten und - als wäre dies nicht genug - mit diesen Daten auch einen gesunden Lebensstil nachweisen konnten (Maturo und Moretti 2018). Längst wurden Daten, insbesondere personalisierte Daten, zum „Rohstoff der Zukunft“, aus deren digitaler Vernetzung nicht nur medizinisch-wissenschaftliche Erkenntnisse zum gesundheitlichen Vorteil der Selftracker, sondern auch unmittelbare Nachteile ökonomischer Art resultieren könnten. Auf die ausführliche Debatte zu Gefahren und Chancen durch Big Data sei an andere Stellen verwiesen (Gethmann 2018; Amunts et al. 2018; UNESCO-ICB 2017; Deutscher Ethikrat 2015, 2017; Mainzer 2014; Stampfl 2013).

\footnotetext{
7 Vgl. etwa: „As these data sources are joined together through Health Information Exchanges (HIEs) and national Electronic Health Records (EHR) systems, a vast scientific dataset spanning millions of individuals will finally be available to health informaticians with the promise of new insights into health and disease" (Paton et al. 2012, S. 18).
} 


\section{Präventive Grundhaltung oder gesellschaftliche Medikalisierung?}

Nun stellt die Datengenerierung und deren Austausch keinen Selbstzweck dar, der in der Auswertung durch Dritte, sei es nun durch die klinische Forschung oder die Ökonomie, ihre Zielbestimmung findet. Gesundheitliches Selftracking zeichnet sich vielmehr in der ermöglichten Evaluation durch den:die Anwender:in selbst aus. Das durch die Vermessung und deren Auswertung erzeugte Gesundheitsbewusstsein der Einzelnen könnte - eine breite Akzeptanz vorausgesetzt - zur präventiven Sensibilisierung der Gesamtgesellschaft beitragen. Durch die technologische Verknüpfung physiologischer Vitaldaten mit statistischen Vergleichsoptionen könne, so die Hoffnung, eine „kompetitive Selbstsorgekultur“ etabliert werden, die beispielsweise im Bereich arbeitsmedizinischer Vorsorge ,den Widerspruch zwischen maximaler Arbeitsleistung und dauerhafter Erhaltung der Arbeitsfähigkeit approximativ als Teil einer technologiegestützten Personalentwicklung zu lösen versucht“ (Mämecke 2016, S. 103). ${ }^{8}$ Aus den mittels Selbstvermessungstechnologien gewonnenen Informationen können durch den Abgleich mit Sozialstatistiken selbstverstärkende Effekte evoziert werden, indem den deskriptiven Daten mögliche Deutungshorizonte und erstrebenswerte Zielvorstellungen im Sinne einer gesundheitlichen Eigenverantwortung anheimgestellt werden. Gesellschaftliches Ziel ist insofern die Befähigung des Nutzenden zu einem gesünderen Lebensstil und Alltagsverhalten (vgl. auch Empowerment im folgenden Kapitel), getragen von der Vision einer möglichst deutlichen Verlagerung weg von der kurativen Therapie bereits Erkrankter hin zur vorrangig präventiven Versorgung noch Gesunder (Maturo und Moretti 2018; UNESCO-ICB 2017; Wiedemann 2016; Johnson et al. 2016). Eine solche präventive Grundhaltung gewann in den letzten Jahrzehnten an deutlicher Relevanz, werden die wirtschaftlichen Ressourcen „westlicher“ Nationen doch v. a. infolge der demographischen Entwicklung durch steigende Fallzahlen Multimorbider und chronisch Erkrankter zusehends herausgefordert (Busse et al. 2010; Maturo und Moretti 2018; Sharon 2017; Thualagant und From 2018). In diesem Sinne ist das individuelle Engagement für die eigene Gesundheit eo ipso auch ein Engagement zur Entlastung des gesellschaftlichen Gesundheitssystems.

Die Verfolgung derartiger Interessen im Sinne des Gemeinwohls bleibt jedoch keineswegs unumstritten. Im Fokus stehen hierbei vorrangig zwei Aspekte: Einerseits wird der von einer Überantwortung im neoliberalen Sinne der Gesundheit an die:den Einzelne:n ausgehende Rückzug des Gesundheitssystems hinsichtlich seiner Verantwortlichkeit als kritisch beäugt (Sharon 2017). Andererseits wird darin abermals eine „Logik der permanenten Leistungssteigerung“ (Duttweiler und Passoth 2016, S. 17) mit der Suggestion einer Notwendigkeit zur etwaigen „Optimierung des Lebens“ (Wiedemann 2016, S. 294; Schaupp 2016, S. 76; Deutscher Ethikrat 2017, etwa S. 120) vermutet, wie sie etwa seit Foucaults Ausführungen zur „Bio-

\footnotetext{
8 Weiter führt dieser exemplarisch am für den AOK-Gesundheitspreis 2013 nominierten Programm ,evergreen“ aus, dieses solle „,neben der Selbsteinschätzung der eigenen Gesundheit zusätzlich externe medizinische Daten (z. B. der Hausärztin) aufnehmen [...], um ,gesundheitshemmende Faktoren` im Unternehmen frühzeitig zu identifizieren, welche die Produktivität mindern und zu hohen Folgekosten führen können“ (Mämecke 2016, S. 104).
} 
macht" in den Vordergrund der anhaltenden Enhancement-Debatte rückte (Foucault 1977; Gehring 2006). Der auf die je eigene Selbstoptimierung ausgerichtete Individualismus werde demzufolge ,zum zentralen Wert der performativen Selbstkontrolle stilisiert“" (Schaupp 2016). Eine derart ausufernde Bedeutung von Gesundheit im Alltag des Einzelnen führe zu einer - man fühlt sich an Ivan Illichs medizinkritische Essays von 1975 erinnert - gesamtgesellschaftlichen „Medikalisierung“ bzw. einem „healthism“ (Maturo und Moretti 2018, S. 47, 51) ${ }^{9}$ mit fragwürdigen Implikationen für den Krankheitsbegriff (Swan 2009).

\section{Individuelles Empowerment oder Kontrollverlust der Patienten?}

Standen bisher die Chancen und Gefahren der Datengenerierung im Allgemeinen sowie die positiven und negativen gesellschaftlichen Implikationen einer zunehmenden Nutzung medizinischen Selftracking im Vordergrund, bedarf es nun noch des Augenmerks auf die Potenziale für die individuelle medizinische Versorgung. Hierbei scheinen sich die bereits angesprochenen Aspekte institutioneller und gesellschaftlicher Art auf der Ebene der:des Einzelnen vor allem im Diskurs um ein Empowerment im Sinne einer gesteigerten Handlungsfähigkeit gegenüber der Gefahr eines Kontrollverlusts zu verdichten.

Das Fundament für eine Förderung der Handlungsfähigkeit besteht im Bereich der Selftracking-Technologien in dem aus den generierten Daten ermöglichten Wissensgewinn über sich und den eigenen Körper und der anzunehmenden dadurch erhöhten Entscheidungskompetenz im Sinne einer gesundheitlichen Selbstsorge. Je mehr Daten den Nutzer:innen zur Verfügung stehen und auf diese Weise sogar bisher unbekannte Aspekte des Körpers aufdecken, desto transparenter seien, so die Hoffnung, die daraus resultierenden Handlungsoptionen für den:die Tracker:in. Die Gesundheit könne auf diese Weise, zumindest sofern sie nicht schon allzu eingeschränkt ist und vertiefter medizinischer Kenntnisse bedürfe, so umfänglich wie möglich der eigenen „Kontrolle“ unterstellt werden (Ruckenstein 2014). „Das Wissen über sich“" werde dabei, so Uwe Vormbusch (2016, S. 55), zur „Voraussetzung von Selbstbestimmung und damit als ein Ziel im Rahmen einer normativen Ordnung qualifiziert, als eine Ressource, um die Fremdbestimmung durch professionelle Akteure (Ärzte beispielsweise) abzustreifen“" (vgl. auch Sharon 2017; Paton et al. 2012; Thualagant und From 2018). Der:die Nutzer:in könne durch DiGA mit personalisierten Informationen zunehmend eine informierte, kollaborative, schlicht aktivere Rolle bezüglich seiner:ihrer eigenen Gesundheit übernehmen. Die so frei gewordenen personellen und institutionellen Kapazitäten könnten eine effizientere und strukturell besser verflochtene Gesundheitsversorgung ermöglichen. Auf das politische Interesse zugunsten einer aktiven Beteiligung angesichts der erwarteten Zukunft des Gesundheitssystems wurde bereits hingewiesen (vgl. bestätigend auch UNESCO-

\footnotetext{
9 Vgl. etwa: „However, if therapy is increasingly important and is at the core of contemporary society and people are a bit sick, pre-sick, or ,at risk', it results in people having to be treated any time; that is why this therapy culture fosters self-tracking, self-monitoring, and self-care. Even when we are not sick at all, we are invited to cultivate preventive habits and try to be ,better than well'“" (Maturo und Moretti 2018, S. 50-51).
} 
ICB 2017; Europäische Kommission 2014). Ein solches Vorgehen kann als aktualisierte Form bereits seit längerem verfolgter gesundheitspolitischer, aber auch medizinischer Selbstsorge-Strategien angesehen werden und stellt keineswegs einen Einschnitt dar, der erst durch Selftracking bzw. DiGA ermöglicht wurde. Bereits in den 1970er-Jahren wurde etwa die heimische Selbstvermessung des Blutdrucks populär und führte auf diese Weise zu einer selbstkontrollierten und eigenverantwortlichen Beteiligung an der eigenen Gesundheit (Duttweiler und Passoth 2016).

Interessanterweise erkennen einige Autor:innen in den Maßnahmen zum vermeintlichen Gewinn an Selbstbestimmung Momente potenziell heteronomer Eingriffe. Der fortwährende kompetitive Vergleich mit dem sozialen Umfeld bzw. Netzwerk stehe der - häufig diskutierten - andauernden digitalen Überwachung durch Politik und Wirtschaft (Big Data) hierbei in nichts nach, sondern bilde mit ihnen gemeinsam ein Panoptikum gesundheitlicher Kontrolle aus. Für sie gehe mit Selftracking daher statt eines Empowerments, vielmehr ein „Disempowerment“ bzw. Verlust von Selbstbestimmung einher (Maturo und Moretti 2018; Sharon 2017; Duttweiler und Passoth 2016; Schaupp 2016). Statistische Maßstäbe und sozialgesellschaftliche Idealtypen, an denen sich die Nutzer auszurichten versuchen, würden die Handlungsfreiheit deutlich eingeschränkt erscheinen lassen, wolle man sich nicht einer gesundheitlichen Diskriminierung aussetzen (Selke 2016a; Maturo und Moretti 2018; Schaupp 2016). Der Deutsche Ethikrat spricht in diesem Sinne von einer „Sogwirkung“, die auf den Nutzer wie ein ,innerer Zwang“ zurückwirken könne (Deutscher Ethikrat 2017). Die normativ anmutende Wirkung kollektiver TrackingDaten auf den Einzelnen führte eindrücklich Alexandra Carmichaels Blogeintrag vor Augen, die nach zweijährigem täglichen Selftracking auf der Plattform „curetogether.com“ ihren Ausstieg beschrieb mit: „What they didn't see/Was/The selfpunishment/The fear/The hatred behind the tracking//I had stopped trusting myself/ Letting the numbers drown out/My intuition/My instinct ${ }^{\text {“ }}{ }^{10}$

Ist also das vorgestellte Ziel des medizinischen Selftracking, möglichst viele Daten und so auch ein „Mehr“ an Wissen zu generieren, tatsächlich vorrangig ent- oder belastend? Das in Selftracking-Technologien steckende Potenzial beinhaltet beides gleichermaßen und bedarf daher, insbesondere bei deren systemisch angestrebter Nutzung innerhalb der Gesundheitsversorgung, einer individuellen Einschätzung der vorgestellten Chancen und Gefahren, die in Abhängigkeit von der jeweiligen Anwendung sowie den Kompetenzen der:des jeweiligen Nutzer:in zu erfolgen hat. Technologien, die die Rezipient:innen durch Edukation zu (partizipativen) Akteur:innen machen, verfolgen letztlich Grundzüge eines gut erprobten und etablierten kognitivverhaltenstherapeutischen Ansatzes. Auf diese Weise durch Selftracking ermöglichte Selbstwirksamkeitserfahrungen wirken sich auch positiv auf das medizinische Outcome aus (Paton et al. 2012). Folglich rückt in das Zentrum des Diskurses um ein ermöglichtes Empowerment sowohl der gesunden wie auch der erkrankten Anwender:innen deren gesundheitsbezogene Reflexions- und Handlungskompetenz (auch Health Literacy bzw. Gesundheitskompetenz) auf Basis der selbstvermessenen Körperdaten. So greift die einseitige Auffassung der Quantified-Self-Avantgar-

10 Vollständiger Eintrag unter: www.quantifiedself.com/blog/why-i-stopped-tracking, zugegriffen: 11. Nov. 2020 (zitiert bei Duttweiler und Passoth 2016). 
de, mittels Kategorien und Ordnungssystemen wie Zahlen werde die Komplexität der Realität strukturiert und vereinfacht (Rode 2019), zu kurz und ist daher nur bedingt korrekt: Einerseits beruhen derartige Ordnungs- und Strukturierungsversuche auf Wahrscheinlichkeiten, die bei fehlenden kontextuellen Zusatzinformationen, wie etwa medizinisch-klinischem Wissen und professioneller Erfahrung, sinken bzw. fehleranfälliger werden. Andererseits kann eine solche Auffassung und mangelnde Kontextualisierung eine ungenügende Einfachheit der Realität suggerieren und auf diese Weise ein vermeintliches Expertentum medizinischer Laien befördern, das mit nachhaltigen Schwierigkeiten für die Kommunikation zwischen Therapeut:innen und Patient:innen einhergehen kann.

Zur Lösung solcher potenziellen Herausforderungen verhilft der Blick in einen bereits seit vielen Jahrzehnten selbstvermessenden Therapiebereich, der an der Förderung der Handlungsfähigkeit Einzelner hinsichtlich ihrer eigenen Gesundheit bzw. Krankheit interessiert ist und nicht zuletzt deshalb Pionierarbeit bei DiGA leistete: die Begleitung von Patient:innen mit chronischen Formen von Diabetes mellitus (hierzu etwa Wiedemann 2016 und Danesi et al. 2018). ${ }^{11}$ Die Erzeugung technisch vermittelter physiologischer Messwerte sowie deren Evaluation gehören nicht erst seit dem Bestehen von Health Apps selbstverständlich zur Therapie des Diabetes: „Blutzucker messen, Broteinheiten anpassen, Gemütszustände aufschreiben, Insulinmengen berechnen oder, nach mehr Bewegung als vorausgesehen, rückrechnen“ (Wiedemann 2016, S. 294). Mithilfe längst etablierter kontinuierlicher Glukosemessung (CGM) durch einen Sensor am Oberarm mit Gewebekontakt und dessen Verknüpfung mit einem Datenprogramm, etwa als Selftracking-App auf dem Smartphone, können sich anbahnende Unterzuckerungen oder andere Schwankungen frühzeitig signalisiert und auch nachträglich evaluiert werden (Wiedemann 2016). Große Teile der alltäglichen diagnostischen und therapeutischen Kompetenz werden hierbei der Eigenverantwortung der Betroffenen übergeben - die alsbald auch ihre individuelle Expertise hinsichtlich ,ihres“ Diabetes gegenüber ihren Ärzt:innen zu verteidigen wissen.

Wie bei anderen Selftracking-Technologien benötigt eine erfolgreiche empowernde Anwendung jedoch über die bloße Bereitstellung von Körperdaten hinaus auch eine individuelle wissensbasierte Anleitung und Begleitung. Die Beförderung eines Wechsels von Rezipient:innen hin zu (partizipativen) Akteur:innen bedarf eben einer expliziten Edukation (Danholt 2013: ,an active patient is not something you are, but something you become“, zitiert in Danesi et al. 2018, S. 132), die - je nach persönlichen Voraussetzungen und Umständen der Betroffenen - äußerst unterschiedlich ausfallen kann. Erfordert der erste Schritt der schlichten Generierung von Daten dank der diversen Health Apps kaum Aufwand durch die selbstvermessende Person, kann der unmittelbar sich anschließende Schritt der Aus- und Bewertung sowie der Einleitung daraus resultierender Handlungen ohne ausreichendes Wissen nicht gelingen. Solches Wissen hat - wie bei jeglicher Form von Therapie bisher - neben medizinischen Fakten hinsichtlich der eigenen Erkrankung auch mögliche Risiken

\footnotetext{
11 Beispielhaft ist hier die App mySugr, die durch Gamifizierung mithilfe eines „Diabetes-Monsters“, das es im Alltag durch das Tracking von Blutzuckerwerten, Ernährung und sportlicher Betätigung zu ,zähmen“ gilt, zur motivationalen Stärkung der Selbstsorge führen soll (Wiedemann 2016).
} 
und Nebenwirkungen zu beinhalten, etwa zu Fehlerraten generierter Wahrscheinlichkeitswerte oder möglichen psychologischen Effekten. Insbesondere eine längerfristige Begleitung einer Selftracking-gestützten präventiven oder kurativen Therapie kann sodann Patient:innen in ihrer gesundheitlichen Handlungsfähigkeit stärken und mit diesen gemeinsam ein aus medizinischer oder psychologischer Sicht erratisches Nutzungsverhalten der Technologien frühzeitig identifizieren.

Keineswegs sollte Empowerment in der Praxis jedoch auf eine bloße ,Vermittlung gesundheitsspezifischen Wissens“ verkürzt werden (Loss 2008, S. 713), in der Hoffnung, bereits durch die Bereitstellung von Informationen „Selbstbestimmung“, „Selbstwirksamkeit“" oder ,Selbstverantwortung“ zu fördern (Pilz et al. 2014). Zwar stellt das diese drei Begriffe verbindende Konzept der gesteigerten Handlungsfähigkeit das vorrangige Ziel des Empowermentprozesses dar. Um dies jedoch tatsächlich erreichen zu können, bedarf es im Rahmen des Empowerment jedoch eben nicht nur einer Befähigung, die beim einzelnen Subjekt ansetzt und dessen Selbstgestaltungkräfte zu födern ersucht, sondern darüber hinaus der Anstrengungen auf überindividueller (Gruppen, Organisationen) und systemischer Ebene (Politik, Gesellschaft), um auf diese Weise ,zu größerer gemeinschaftlicher Stärke und Handlungsfähigkeit“" zu führen (Stark und Brandes 2016) und eine gesamtgesellschaftliche Wirkung zu erzielen. Für die Implementierung von DiGA im Allgemeinen, aber auch angesicht der hier geschilderten großen Potenziale von Selftracking-Anwendungen im Speziellen stellt dies folgende Herausforderung dar: Nebst der anfänglichen und - wo dies sinnvoll erscheint - fortwährenden therapeutisch-individuellen Bereitstellung von Begleitungsmöglichkeiten in der Nutzung von Gesundheitsanwendungen gerät insbesondere die Schaffung institutioneller Settings und methodischer Verfahren zur anhaltenden Befähigung in das Zentrum der Aufmerksamkeit. Insofern stellt Empowerment eine strukturelle Forderung zur Erreichung patient:innen- und nutzer:innenzentrierter Formen des Krankheits- und Gesundheitsmanagements dar.

\section{Implikationen für Health Apps in der medizinischen Regelversorgung}

Mit Inkrafttreten des Digitale-Versorgung-Gesetzes (DVG) am 19. Dezember 2019 können erstmals DiGA als Medizinprodukte auf Rezept verschrieben werden ( $\$ 33 \mathrm{a}$ SGB V). Dem Gesetz zufolge handelt es sich dabei um Medizinprodukte niedriger Risikoklasse (I oder IIa), ,deren Hauptfunktion wesentlich auf digitalen Technologien beruht und die dazu bestimmt sind, bei den Versicherten oder in der Versorgung durch Leistungserbringer die Erkennung, Überwachung, Behandlung oder Linderung von Krankheiten oder die Erkennung, Behandlung, Linderung oder Kompensierung von Verletzungen oder Behinderungen zu unterstützen." Voraussetzung für die Verordnung einer DiGA durch behandelnde Ärzt:innen oder Psychotherapeut:innen ist die Aufnahme der Anwendung in ein neu zu schaffendes, vom Bundesinstitut für Arzneimittel und Medizinprodukte (BfArM) geführtes Verzeichnis (vgl. § 139e SGB V), dessen Rechtsverordnung zur Festlegung näherer Konditionen sich aktuell noch in der Entwurfsphase befindet (Stand: Sommer 2020).

Wenngleich basierend auf ersten Entwürfen intensiv über die Gewährleistung datenschutzrechtlicher Minimalvoraussetzungen und deren Überprüfung (vgl. Big Da- 
ta) diskutiert ${ }^{12}$ und über mögliche Verfahren des Nachweises eines „,medizinischen Nutzens“ oder eines „Beitrags zur Verbesserung der Versorgungsstruktur“, die unter dem Begriff „Positive Versorgungseffekte“ zusammengefasst werden könnten, nachgedacht wird (BVMed 2019, passim), geben diese Entwürfe angesichts des vielschichtigen Diskurses bereits der hier dargestellten Ausführungen Anhalt zur Skepsis. Während unter dem Stichwort „Realistische Evaluationskonzepte“ mittels eines Fast-Track-Verfahrens bisherige Evidenz-Standards für Medizinprodukte, allen voran dem Nutzennachweis durch randomisierte kontrollierte Studien (RCT), ausgeschlagen werden, da diese für Produkte wie DiGA mit ihrer hohen Agilität und einem schnellen Lebenszyklus eine ,kaum zu überwindende Hürde“ darstellen würden, und es darüber hinaus sogar ermöglicht werden soll, Nachweise auch erst bis zu zwölf Monate nach Aufnahme in das Verzeichnis nachreichen zu können (BVMed 2019, S. 2), somit also vorrangig Interessen der Health Apps-entwickelnden Unternehmen vertreten werden, erfahren die Informationsbedarfe der Patient:innen und Therapeut:innen - abgesehen von der strategischen Positionierung an der ersten Stelle des Positionspapiers - verhältnismäßig wenig Aufmerksamkeit.

Durch das frei zugängliche Verzeichnis solle man zwar einen Überblick über die zahlreichen Versorgungsoptionen mit DiGA erhalten, zu klären wäre hierbei jedoch, worin dieser Überblick konkret bestehen soll. Neben Informationen zu passenden medizinischen Indikationen sollten auch Risiken der Anwendungen explizit benannt werden. Zwar erfordern auch DiGA als reguläre Medizinprodukte den sog. Facharztstandard, demnach Ärzt:innen ihren Patient:innen ausreichende Informationen auch hinsichtlich des Gefahrenpotentials schulden (Fehleranfälligkeit, psychologische Effekte). Inwiefern aber tatsächlich diese Informationen angesichts der unüberschaubaren und weiter anwachsenden Fülle an DiGA genügen, damit Nutzer:innen durch die eigenständige Kontextualisierung und Evaluation der Daten einen möglichst hohen Mehrwert erzeugen und möglichst keine Formen des Kontrollverlusts erleben, bleibt angesichts eines lediglich an vermeintlich positiven Aspekten orientierten Verzeichnisses fraglich. Wünschenswert wäre hierbei folglich eine ausgewogene Darstellung möglicher positiver und negativer Potenziale sowie kontextualisierender Informationen einzelner Anwendungen, die jedoch um ein evidenzbasiertes Evaluationskonzept nicht umhin kämen. Nur mittels eines solchen Weges können jedoch bestmögliche Erfolge hinsichtlich eines Empowerment der Patient:innen erzielt sowie gegenläufige Tendenzen, etwa im Sinne eines Kontrollverlusts, verhindert werden. Aufgrund dieses Mangels werden auch potenzielle Chancen hinsichtlich einer durch DiGA gestärkten präventiven Grundhaltung verkannt, bedarf auch sie der gesundheitlich befähigten Nutzer:innen.

Die Faszination an und die Suche nach einer Perfektion des menschlichen Körpers und damit einhergehend Selbstvermessungen begleiten die Geschichte seit langem, wie dies etwa Leonardos homo vitruvianus deutlich vor Augen führt. Naheliegend erhielten derartige Selbstvermessungs-Praktiken eine hohe Relevanz für die Medizin, bieten diese nicht nur Chancen einer Förderung (patho-)physiologischer Erkenntnis-

\footnotetext{
12 Hierbei wird etwa eine CE-Zertifizierung als zu erfüllender Grundstandard von Leistungsfähigkeit und Sicherheit geltend gemacht; vgl. hierzu die Ausführungen des Bundesverbands für Medizintechnologie (BVMed 2019).
} 
se, sondern auch Potenziale im Sinne einer gesundheitlichen Eigenverantwortung und Kompetenz - insofern handlungsfähigerer Nutzer:innen. Dieses Ziel bedarf jedoch neben der Vermittlung von Grenz- und Normwerten auch der Bereitstellung kontextualisierender Zusatzinformationen und einer den Adressat:innen angemessenen Vermittlung, die die Deutungshoheit von Krankheit und Gesundheit nicht allein statistischen Datensätzen überlässt. Die Implementierung von DiGA im deutschen Gesundheitswesen birgt zahlreiche der oben angeführten Potenziale, von einer Steigerung der individuellen gesundheitlichen Selbstsorge über eine gesamtgesellschaftliche präventive Grundhaltug bis hin zu gegebenenfalls ermöglichten empirischen Erkenntnisgewinnen. Um hierbei jedoch die positiven Potenziale unter möglichst weitreichendem Ausschluss der negativen Potenziale umfassend zu verwirklichen, erfordert die Implementierung ein Empowerment der Nutzer:innen sowohl auf der individuellen als auch durch die Bereitstellung geeigneter Settings und Verfahren auf der überindividuellen und systemischen Ebene.

Funding Open Access funding enabled and organized by Projekt DEAL.

Open Access Dieser Artikel wird unter der Creative Commons Namensnennung 4.0 International Lizenz veröffentlicht, welche die Nutzung, Vervielfältigung, Bearbeitung, Verbreitung und Wiedergabe in jeglichem Medium und Format erlaubt, sofern Sie den/die ursprünglichen Autor(en) und die Quelle ordnungsgemäß nennen, einen Link zur Creative Commons Lizenz beifügen und angeben, ob Änderungen vorgenommen wurden.

Die in diesem Artikel enthaltenen Bilder und sonstiges Drittmaterial unterliegen ebenfalls der genannten Creative Commons Lizenz, sofern sich aus der Abbildungslegende nichts anderes ergibt. Sofern das betreffende Material nicht unter der genannten Creative Commons Lizenz steht und die betreffende Handlung nicht nach gesetzlichen Vorschriften erlaubt ist, ist für die oben aufgeführten Weiterverwendungen des Materials die Einwilligung des jeweiligen Rechteinhabers einzuholen.

Weitere Details zur Lizenz entnehmen Sie bitte der Lizenzinformation auf http://creativecommons.org/ licenses/by/4.0/deed.de.

\section{Einhaltung ethischer Richtlinien}

Interessenkonflikt F. Funer gibt an, dass kein Interessenkonflikt besteht.

Ethische Standards Für diesen Beitrag wurden vom Autor keine Studien an Menschen oder Tieren durchgeführt. Für die aufgeführten Studien gelten die jeweils dort angegebenen ethischen Richtlinien.

\section{Literatur}

Abend P, Fuchs M, Reichert R, Wenz K (2016) Quantified selves and statistical bodies. transcript, Bielefeld Amunts K, Klingmüller U, Bormann F-J (2018) Big Data in der Grundlagenforschung und der medizinischen Anwendung. ZfmE 64:99-118

Bitkom Research (2019) Präsentation „Digital Health“ Achim Bergs am 08.05.2019 in Berlin. www. bitkom.org/sites/default/files/2019-05/190508_bitkom-pressekonferenz_e-health_prasentation.pdf.

Zugegriffen: 11. Nov. 2020

Bundesgesetzblatt (2019) I, Nr. 49, augegeben am 18. Dezember 2019, S 2562-2584

Bundesverband für Medizintechnologie BVMed (2019) Positionspapier zum Verzeichnis für DiGA nach $\S 139$ e SGB V vom 27.11.2019. www.bvmed.de/dvg-positionspapier. Zugegriffen: 11. Nov. 2020

Busse R, Blümel M, Scheller-Kreinsen D, Zentner A (2010) Tackling chronic disease in Europe. Strategies, interventions and challenges. WHO, Copenhagen 
Danesi G, Pralong M, Pidoux V (2018) Embodiment and agency through self-tracking practices of people living with diabetes. In: Ajana B (Hrsg) Metric culture. Ontologies of self-tracking practices. Emerald, Bingley, S 117-136

Depper A, Howe PD (2017) Are we fit yet? English adolescent girls' experiences of health and fitness apps. Health Sociol Rev 26:98-112

Deutscher Ethikrat (2015) Simultanmitschrift der Jahrestagung „Die Vermessung des Menschen - Big Data und Gesundheit" am 21.05.2015. www.ethikrat.org/fileadmin/PDF-Dateien/Veranstaltungen/jt21-05-2015-simultanmitschrift.pdf. Zugegriffen: 11. Nov. 2020

Deutscher Ethikrat (2017) Big Data und Gesundheit - Datensouveränität als informationelle Freiheitsgestaltung. Stellungnahme. Deutscher Ethikrat, Berlin

Didžiokaite G, Saukko P, Greiffenhagen C (2018) The mundane experience of everyday calorie trackers: beyond the metaphor of quantified self. New Media Soc 20:1470-1487

Duttweiler S, Passoth J-H (2016) Self-Tracking als Optimierungsprojekt? In: Duttweiler S, Gugutzer R, Passoth J-H, Strübing J (Hrsg) Leben nach Zahlen. Self-Tracking als Optimierungsprojekt? transcript, Bielefeld, S 9-44

Duttweiler S, Gugutzer R, Passoth J-H, Strübing J (Hrsg) (2016) Leben nach Zahlen. Self-Tracking als Optimierungsprojekt? transcript, Bielefeld

Elsden C, Kirk D (2014) A quantified past: remembering with personal informatics. In: Wakkary R, Harrison S (Hrsg) DIS Companion '14: Proceedings of the 2014 companion publication on Designing interactive systems, S 45-48 https://doi.org/10.1145/2598784.2602778

Europäische Kommission (2014) Grünbuch über Mobile-Health-Dienst (,mHealth“). Europäische Kommission, Brüssel

Flügel B, Greil H, Sommer K (1986) Anthropologischer Atlas. Grundlagen und Daten. Alters- und Geschlechtsvariabilität des Menschen. Wötzel, Frankfurt a. M.

Fotopoulou A, O'Riordan K (2017) Training to self-care: Fitness tracking, biopedagogy and the healthy consumer. Health Sociol Rev 26:54-68

Foucault M (1977) Überwachen und Strafen. Suhrkamp, Frankfurt a. M.

Gehring P (2006) Was ist Biomacht? Vom zweifelhaften Mehrwert des Lebens. Campus, Frankfurt a. M.

Gethmann C-F (2018) Ethische Überlegungen zu den Chancen und Risiken der digitalen Agenda im Gesundheitssystem. ZfmE 64:87-97

Harrison V et al (2011) Mobile mental health: review of the emerging field and proof of concept study. J Ment Health 20:509-524

International Bioethics Committee (IBC) of UNESCO (2017) Report of the IBC on big data and health. unescoblob.blob.core.windows.net/pdf/UploadCKEditor/REPORT\%20OF\%20THE\%20IBC \%20ON\%20BIG\%20DATA\%20AND\%20HEALTH\%20\%2015.09.17.pdf. Zugegriffen: 11. Nov. 2020

Jethani S (2015) Mediating the body. Technology, and epistemologies of self. Commun Polit Cult 47:34-43

Johnson D et al (2016) Gamification for health and wellbeing: a systematic review of the literature. Internet Interv 6:89-106

Laakso E, Armstrong K, User W (2011) Cyber-management of people with chronic disease: a potential solution to eHealth challenges. Health Educ J 5:1-8

Link J (2006) Versuch über den Normalismus. Wie Normalität produziert wird. Vandenhoeck \& Ruprecht, Göttingen

Loss J (2008) Der Empowerment-Ansatz: unscharf, unbequem, unberechenbar - und unentbehrlich. Gesundheitswesen 70:713-714

Lupton D (2013) Quantifying the body: monitoring and measuring health in the age of mhealth technologies. Crit Public Health 23:393-403

Lupton D (2014) Critical perspectives on digital health technologies. Soc Comp 8:1344-1359

Lupton D (2016) The quantified self. A sociology of self-tracking. Polity Press, Cambridge (UK)

Mainzer K (2014) Die Berechnung der Welt. Von der Weltformel zu Big Data. C. H. Beck, München

Mämecke T (2016) Benchmarking the Self. Kompetitive Selbstvermessung im betrieblichen Gesundheitsmanagement. In: Duttweiler S, Gugutzer R, Passoth J-H, Strübing J (Hrsg) Leben nach Zahlen. SelfTracking als Optimierungsprojekt? transcript, Bielefeld, S 103-122

Mämecke T, Passoth J-H, Wehner J (2018) Bedeutende Daten. Modelle, Verfahren und Praxis der Vermessung und Verdatung im Netz. Springer VS, Wiesbaden

Maturo A, Moretti V (2018) Digital health and the gamification of life. How apps can promote a positive medicalization. Emerald, Bingley

Nafus D, Sherman J (2014) This one does not go up to 11: the quantified self movement as an alternative big data practice. Int J Commun 8:1784-1794 
Pantzar M, Ruckenstein M (2015) The heart of everyday analytics: emotional, material and practical extensions in self-tracking market. Consump Mark Cult 18:92-109

Paton C, Hansen M, Fernandez-Luque L, Lau AYS (2012) Self-tracking, social media and personal health records for patient empowered self-care. Yearb Med Inform 7:16-24

Pilz W, Brock I, Thiemann J (2014) Einführung. In: Brock I (Hrsg) Psychotherapie und Empowerment Impulse für die psychosoziale Praxis. Verlag Barbara Budrich, Leverkusen-Opladen, S 9-18

Rabin C, Bock R (2011) Desired features of smartphone applications promoting physical activity. Telemed E-health 17:801-803

Reichert R (2016) Social Surveillance. Praktiken der digitalen Selbstvermessung in mobilen Anwendungskulturen. In: Duttweiler S, Gugutzer R, Passoth J-H, Strübing J (Hrsg) Leben nach Zahlen. SelfTracking als Optimierungsprojekt? transcript, Bielefeld, S 185-200

Rode D (2019) Selbst-Bildung im und durch Self-Tracking. Ein analytisch-integrativer Systematisierungsversuch zur Subjektkultur des ,,neuen Spiels“ digitaler Selbstvermessung. In: Rode D, Stern M (Hrsg) Self-Tracking, Selfies, Tinder und Co. Konstellationen von Körper, Medien und Selbst in der Gegenwart. transcript, Bielefeld, S 151-182

Ruckenstein M (2014) Visualized and interacted life. Personal analytics and engagement with data doubles. Societies 4:68-84

Schaupp S (2016) „Wir nennen es flexible Selbstkontrolle.“ Self-Tracking als Selbsttechnologie des kybernetischen Kapitalismus. In: Selke S (Hrsg) Lifelogging. Digitale Selbstvermessung und Lebensprotokollierung zwischen disruptiver Technologie und kulturellem Wandel. Springer, Wiesbaden, S 63-86

Selke S (2016a) Ausweitung der Kampfzone. Rationale Diskriminierung durch Lifelogging und die neue Taxonomie des Sozialen. In: Selke S (Hrsg) Lifelogging. Digitale Selbstvermessung und Lebensprotokollierung zwischen disruptiver Technologie und kulturellem Wandel. Springer, Wiesbaden, S 309-340

Selke S (Hrsg) (2016b) Lifelogging. Digitale Selbstvermessung und Lebensprotokollierung zwischen disruptiver Technologie und kulturellem Wandel. Springer, Wiesbaden

Sharon T (2017) Self-tracking for health and the quantified self: re-articulating autonomy, solidarity, and authenticity in an age of personalized healthcare. Philos Technol 30:93-121

Stampfl N (2013) Die berechnete Welt. Leben unter dem Einfluss von Algorithmen. Heise, Hannover

Stark W, Brandes S (2016) Empowerment/Befähigung. BZgA-Leitbegriffe, Stand vom 9. Februar 2016 https://doi.org/10.17623/BZGA:224-i010-1.0

Steinhubl S, Muse E, Topol E (2013) Can mobile health technologies transform health care? JAMA 310:2395-2396

Swan M (2009) Emerging patient-driven health care models: an examination of health social networks, consumer personalized medicine and quantified self-tracking. Int J Environ Res Publ Health $6: 492-525$

Thualagant N, From D-M (2018) The digitisation of welfare: a strategy towards improving citizens' selfcare and co-management of welfare. In: Ajana B (Hrsg) Metric culture. Ontologies of self-tracking practices. Emerald, Bingley, S 37-56

Till C (2014) Exercise as labour: quantified self and the transformation of exercise into labour. Societies 4:446-462

Unternährer M (2016) Selbstquantifizierung als numerische Form der Selbstthematisierung. In: Duttweiler S, Gugutzer R, Passoth J-H, Strübing J (Hrsg) Leben nach Zahlen. Self-Tracking als Optimierungsprojekt? transcript, Bielefeld, S 201-220

Villa P-I (2012) Die Vermessung des Selbst. Einsicht in die Logik zeitgenössischer Körperarbeit. Aviso 3:14-19

Vitruv (1964) De architectura libri decem. Wissenschaftliche Buchgesellschaft, Darmstadt

Vormbusch U (2016) Taxonomien des Selbst. Zur Hervorbringung subjektbezogener Bewertungsordnungen im Kontext ökonomischer und kultureller Unsicherheit. In: Duttweiler S, Gugutzer R, Passoth J-H, Strübing J (Hrsg) Leben nach Zahlen. Self-Tracking als Optimierungsprojekt? transcript, Bielefeld, S 45-62

Whitson J (2013) Gaming the quantified self. Surveill Soc 11:163-176

Wiedemann L (2016) ,,Vom Piksen zum Scannen, vom Wert zu Daten.“ Digitalisierte Selbstvermessung im Kontext Diabetes. In: Duttweiler S, Gugutzer R, Passoth J-H, Strübing J (Hrsg) Leben nach Zahlen. Self-Tracking als Optimierungsprojekt? transcript, Bielefeld, S 293-324

Wolf G (2010) „The data-driven life“. The New York Times vom 02.05.2010, S 38. www.nytimes.com/ 2010/05/02/magazine/02self-measurement-t.html. Zugegriffen: 11. Nov. 2020 
Zillien N, Fröhlich G, Dötsch M (2015) Zahlenkörper. Digitale Selbstvermessung als Verdinglichung des Körpers. In: Hahn K, Stempfhuber M (Hrsg) Präsenzen 2.0. Körperinszenierung in Medienkulturen. Springer, Wiesbaden, S 77-94

Zimmermann A (Hrsg) (1984) Mensura. Maß, Zahl, Zahlensymbolik im Mittelalter. De Gruyter, Berlin, New York 